\title{
A comunidade de abelhas (Hymenoptera, Apoidea) em áreas florestais do Parque Estadual de Itapuã (Viamão, RS): diversidade, abundância relativa e atividade sazonal
}

\author{
Betânia Truylio' ${ }^{1} \&$ Birgit Harter-Marques ${ }^{2}$
}

1. Laboratório de Pesquisas Biológicas, Faculdade de Biociências, Pontifícia Universidade Católica do Rio Grande do Sul (PUCRS), Av. Ipiranga, 6681, 90619-900 Porto Alegre, RS, Brasil. (btruylio@pucrs.br)

2. Laboratório de Abelhas Silvestres, Programa de Pós-Graduação em Ciências Ambientais, Universidade do Extremo Sul Catarinense (UNESC), Av. Universitária, 1105, 88806-000 Criciúma, SC, Brasil. (bhm@unesc.net)

\begin{abstract}
The bee community (Hymenoptera, Apoidea) of forest areas of Parque Estadual Itapuã (Viamão, Southern Brazil): diversity, relative abundance and seasonal activity. The bee community of Parque Estadual de Itapuã, Viamão, Southern Brazil, was studied regarding diversity, relative abundance and seasonal activity. The bees were collected on the flowers in two forest areas, during two consecutive days respectively, twice per month, from 8 a.m. to 6 p.m., during one year. A total of 3,306 bees from 95 species and five families occurring in Brazil were captured. Among them, Apidae was the most abundant (2,860 individuals) and the most diverse family (36 species), followed by Halictidae, with 308 individuals and 26 species. Comparisons with faunas of other communities revealed a lower diversity at our study site. Bees of the families Apidae and Halictidae remained active throughout the year, with a gradual reduction of activity in March and April, when the lowest index of rainfall and the highest temperature were recorded.
\end{abstract}

KEYWORDS. Bees, phenology, species richness.

RESUMO. A comunidade de abelhas (Hymenoptera, Apoidea) foi estudada no Parque Estadual do Itapuã, município de Viamão, no que diz respeito à diversidade, abundância relativa e atividade sazonal. As abelhas foram coletadas sobre as flores em duas áreas florestais, durante dois dias consecutivos por área, quinzenalmente, das $8 \mathrm{~h}$ às $18 \mathrm{~h}$. No total, foram capturadas 3.306 abelhas pertencentes a 95 espécies das cinco famílias ocorrentes no Brasil. Dentre elas, Apidae foi a família mais abundante (2.860 indivíduos) e mais diversificada (36 espécies), seguida de Halictidae com 308 indivíduos e 26 espécies. Comparações realizadas com a fauna de abelhas de outras comunidades revelaram uma baixa diversidade na região estudada. Representantes de Apidae e Halictidae foram encontrados o ano todo nas flores, com uma redução gradual de atividade em março e abril quando foi registrado o menor índice de precipitação e temperaturas mais altas do ano.

PALAVRAS-CHAVE. Abelhas, fenologia, riqueza.

Cada vez mais as abelhas vêm sendo valorizadas pelo importante papel que cumprem nas comunidades ecológicas. As abelhas constituem os principais agentes polinizadores adaptados à visita às flores de Angiospermas, provendo e aumentando a produção de frutos comestíveis para o homem e muitos animais silvestres (WiLLiams et al., 1991). Além disso, auxiliam na produção de sementes para a reprodução das plantas, mantendo a diversidade do ecossistema (SILVEIRA et al., 2002).

Estima-se que existam mais de quatro mil gêneros e cerca de 25 a 30 mil espécies de abelhas distribuídas nas diferentes regiões do mundo (MichenER, 2000). Cerca de 85\% dessas espécies são solitárias (BATRA, 1984) e muitas delas ainda não foram descritas, principalmente nos trópicos. No Brasil, SiLveIra et al. (2002) relacionam 1.573 nomes válidos para as espécies brasileiras, mas acreditam que a fauna de abelhas do Brasil seja composta por, aproximadamente, 3.000 espécies.

Os levantamentos melissofaunísticos em áreas restritas, por meio de amostragens padronizadas, tiveram início com o trabalho de SAKAGAMI \& Matsumura (1967), no Japão e com o trabalho de SAKAGAMI et al. (1967), no sul do Brasil. Até essa época, poucas eram as contribuições sobre a ecologia de comunidades de visitantes florais e essas eram realizadas por meio de procedimentos parcialmente padronizados (COCKERELL, 1900; SCHROTTKY, 1902; DUCKE, 1906, 1907; FRIESE, 1910; DUCKE, 1925).
A partir da década de 70 , vários levantamentos sobre a fauna de abelhas e a relação com a flora foram realizados em diferentes ecossistemas, mostrando que o estudo dessas comunidades representa uma abordagem adequada na procura de padrões de organização destas. No Brasil, existem inúmeros levantamentos da apifauna e flora apícola que mostram a importância das plantas na dieta e manutenção das populações das abelhas, assim como a importância das abelhas no processo de polinização das plantas que visitam (e.g., SAKAGAMI \& LAROCA, 1971; CAMARGO \& MAZUCATO, 1984; GotTSBERGER et al., 1988; Viana et al., 1997; AgUiar, 2003; ANTONINI \& MARTINS, 2003).

O Estado do Rio Grande do Sul, apesar do seu tamanho territorial relativamente pequeno, apresenta uma grande diversidade de ecossistemas (RADAMBRASIL, 1981). Até o momento, estudos sobre a fauna de abelhas e a flora apícola associada no estado foram realizados na região da Serra do Sudeste (Schlindwein, 1995), no litoral (Alves-dos-Santos, 1999) e em diversas regiões fitogeográficas por WitTMANN \& HofFMANN (1990). Todos estes levantamentos têm sido realizados em ambientes relativamente conservados e estudos de interação abelhaplanta em regiões metropolitanas inexistem.

O Parque Estadual de Itapuã é uma unidade de conservação integral que apresenta a maior diversidade de ecossistemas entre os parques estaduais do Rio 
Grande do Sul, sendo esta área considerada última amostra dos ecossistemas originais como a da Região Metropolitana de Porto Alegre. Contudo, a vegetação do Parque encontra-se em diferentes estados de regeneração devido à grande pressão antrópica exercida no passado (Rio Grande do Sul, 1997). Iniciaram-se alguns estudos no Parque com o objetivo de preservar e regenerar o ecossistema original, principalmente sobre a composição florística da região. Porém, nada se sabe sobre a estrutura de comunidades de visitantes florais, ainda que tal conhecimento seja essencial tanto para a manutenção de áreas fragmentadas (BAwA, 1990) quanto para programas de manejo em unidades de conservação. Com efeito, o levantamento e a identificação das espécies de abelhas constituem o primeiro passo para se conhecer os polinizadores e definir estratégias de exploração racional e conservação dos recursos biológicos encontrados nas comunidades de vegetais e de animais (KeVAN \& BAKER, 1983; Matheson et al., 1996; Proctor et al., 1996).

Diante disso, o presente trabalho teve como objetivo contribuir para o conhecimento da diversidade de abelhas no estado, assim como estimar a abundância e a atividade sazonal destes insetos em áreas florestais do Parque Estadual de Itapuã.

\section{MATERIAL E MÉTODOS}

As coletas foram realizadas no Parque Estadual de Itapuã (PEI), localizado ao sul do Distrito de Itapuã, no município de Viamão, Rio Grande do Sul, Brasil, entre as coordenadas $30^{\circ} 20^{\prime} \mathrm{S}$ e $51^{\circ} 05^{\prime} \mathrm{W}$. A área total do Parque abrange aproximadamente 5.500 ha, tendo como limites ao norte o Hospital de Itapuã e o Beco Santa Fé, ao sul e a leste a laguna dos Patos e a oeste o lago Guaíba. As altitudes variam de 5 a 263 m, estando as formas de relevo associadas às duas principais províncias geomorfológicas do estado: os granitos do Escudo Sul-rio-grandense e os sedimentos da Planície Costeira (Rio Grande do Sul, 1997).

O clima local classifica-se como subtropical úmido, sem estação seca, com temperaturas médias anuais oscilando em torno de $17,5^{\circ} \mathrm{C}$ e precipitação média anual entre 1.100 a $1.300 \mathrm{~mm}$. As chuvas são bem distribuídas ao longo do ano e os verões quentes, com temperatura média do mês mais quente superior a $22^{\circ} \mathrm{C}$ (RIo GRANDE Do SuL, 1997). Os dados climáticos registrados durante o período de estudo foram obtidos na Seção de Observação e Meteorologia Aplicada (SEOMA) do Oitavo Distrito de Meteorologia ( $8^{\circ}$ DISME) do Instituto Nacional de Meteorologia (INMET), referentes à cidade de Porto Alegre, distante $57 \mathrm{~km}$ da área de estudo.

A vegetação do PEI apresenta uma cobertura muito diversificada, formada principalmente pelos seguintes tipos fisionômicos: floresta, campo rupestre, mata de restinga, vassoural, campo misto, maricazal e juncal (RIO Grande do Sul, 1997). As formações florestais apresentam composição de Floresta Estacional Semidecidual (TeIXeIRA et al., 1986; PALMA \& JARENKOW, 2003) e Floresta Ombrófila Densa, denominada simplesmente por floresta alta (BRACK et al., 1998), ou por Floresta Ombrófila Densa Submontana de solos profundos (PORTO \& MELLO, 1998).
Para verificar se existe uma influência do grau de degradação antrópica na composição da apifauna, foram escolhidas duas áreas florestais para a realização do estudo. As duas áreas estão classificadas, segundo o plano de manejo, em duas diferentes zonas de conservação. A primeira área está incluída na chamada Zona Primitiva, a qual contém espécies da fauna e da flora de grande valor científico e onde ocorreu pequena ou mínima intervenção humana. Está situada na encosta oeste de um morro, o Morro da Grota, num trecho contínuo de mata formado por árvores não muito altas.

A segunda área está incluída na Zona Primitiva e na Zona de Uso Extensivo, constituída em sua maior parte por áreas naturais, porém podendo apresentar alguma alteração humana. Localiza-se ao longo de uma trilha (Trilha da Onça), utilizada pelos visitantes para passeios ecológicos, e áreas adjacentes. Pela proximidade da praia, a vegetação desta área é típica de mata ciliar com afloramentos rochosos (RIO GRANDE Do Sul, 1997).

As unidades de amostragem nas duas áreas florestais foram demarcadas e instaladas de modo semipermanente. Para cada área foi estabelecido um transecto amostral de aproximadamente $500 \mathrm{~m}$ de comprimento por $2 \mathrm{~m}$ de largura, totalizando $1.000 \mathrm{~m}^{2}$ ao longo de trilhas pré-existentes e nas bordas da mata.

As amostragens foram realizadas quinzenalmente de agosto/2003 a julho/2004, durante dois dias consecutivos para cada área no período das $8 \mathrm{~h}$ às $18 \mathrm{~h}$. Os transectos foram percorridos a passo lento e todas as plantas encontradas em flor foram examinadas em busca de insetos. Nas plantas, independente da quantidade de flores ou visitantes, o coletor permaneceu exatamente 10 minutos. Após ter percorrido o transecto, voltou-se ao ponto inicial e realizou-se uma nova seqüência de coleta, totalizando três sequiências de coleta por dia em cada área. No segundo dia da coleta, realizou-se o percurso inverso ao do dia anterior.

Os visitantes foram capturados com rede entomológica, individualmente ou em grupo, quando pousavam nas flores ou logo após abandoná-las. Para a coleta nas copas das árvores, utilizou-se uma rede com cabo regulável que alcançava até $9 \mathrm{~m}$ de altura. Os insetos coletados foram colocados em câmaras mortíferas com acetato de etila, devidamente numerados com o nome ou número da planta visitada, o horário e a data de coleta.

Paralelamente às coletas nas flores, foram utilizadas armadilhas para amostragem das espécies de Euglossina (Hymenoptera, Apidae) presentes na área. As armadilhas utilizadas foram confeccionadas conforme CAMPOS et al. (1989), com modificações, utilizando-se como compostos odoríferos escatol e $\beta$-ionone.

Em laboratório, os espécimes foram montados, identificados e depositados na coleção de Insecta do Museu de Ciências e Tecnologia na Pontifícia Universidade Católica do Rio Grande do Sul. Nas identificações, seguiu-se a nomenclatura de SilveIRA et al. (2002). As morfoespécies foram comparadas com os indivíduos da coleção de Insecta na PUCRS e devidamente numeradas.

Para comparar a diversidade e a eqüitatividade das espécies de abelhas coletadas nas flores com outros estudos do Brasil que utilizaram a mesma metodologia de 
coleta, usou-se o índice de Shannon-Wiener (KREBS, 1998), desconsiderando as espécies de Euglossina. A distribuição do número de indivíduos por espécie foi analisada segundo o método de PrESTON (1948), que consiste na distribuição gráfica da freqüência das espécies por classes de número de indivíduos (oitavas de abundância). Este método permite visualizar a riqueza e a distribuição quantitativa dos indivíduos por espécie (KREBS, 1998; VIANA, 1999).

\section{RESULTADOS E DISCUSSÃO}

Diversidade e abundância relativa. Durante o período de estudo, um total de 3.306 abelhas de 95 espécies, 38 gêneros e cinco famílias foram coletadas (Tab. I). A homogeneidade de abelhas do Parque, quando comparada à de outros estudos no Brasil, é baixa $\left(\mathrm{H}^{\prime}=\right.$ $\left.2,09, \mathrm{~J}^{\prime}=0,46\right)$, sendo superior apenas ao estudo realizado no Estado de Tocantins por SANTOs et al. (2004) (H' = $\left.1,55, \mathrm{~J}^{\prime}=0,35\right)$ (Tab. II).

O baixo índice de eqüitatividade encontrado é decorrente do grande número de espécies com poucos indivíduos e de poucas espécies com muitos indivíduos, tais como Apis mellifera Linnaeus, 1758, Plebeia emerina (Friese, 1900) e Trigona spinipes (Fabricius, 1793), observado pela distribuição das espécies em classes de abundância (Fig. 1). A curva $\log N$ ajustada para os dados do Parque resultou numa curva truncada, com a extremidade esquerda incompleta (Fig. 1). A localidade estudada apresenta grande parte das espécies situada nas primeiras três classes de abundância (1-7 indivíduos) e um declínio mais intenso na quantidade de espécies a partir da quarta classe. Este grande número de espécies representadas por poucos exemplares, revela uma tendência à presença de espécies raras, sendo que $46 \%$ das espécies estão incluídas nas primeiras duas classes de abundância (até três indivíduos) (Fig. 1). Um padrão semelhante foi obtido por LAROCA (1992), a partir da análise dos padrões de distribuição de densidade em diversas associações de abelhas das regiões Holártica e Neotropical. Segundo Preston (1948), distribuições truncadas refletem o fato de que as amostras não são representativas da totalidade de espécies da comunidade investigada.

Do total de indivíduos coletados, 2.227 abelhas de 77 espécies foram coletadas na área 1 e 1.079 abelhas de 52 espécies na área 2 (Tab. III). Como o tamanho das áreas e o esforço de coleta foram iguais em cada uma delas, o número de indivíduos registrados representa diretamente a densidade de abelhas em cada área. Por conseguinte, a densidade de abelhas na área 1 mostrouse cerca de duas vezes maior do que na área 2.

Das 95 espécies, 34 (35,8\%) foram coletadas em ambas as áreas, 43 espécies $(45,2 \%)$ foram registradas apenas na área 1 e $18(19 \%)$ apenas na área 2 . A diversidade de espécies na área 1 foi mais heterogênea em termos de abundância do que na área $2\left(\mathrm{H}^{\prime}=2,07 \mathrm{e}\right.$ 1,$71 ; \mathrm{J} '=0,47$ e 0,43 respectivamente). Esta diferença observada tanto na abundância quanto na riqueza de abelhas nas duas áreas de estudo, provavelmente está relacionada ao tipo de vegetação e ao grau de alteração antrópica de cada área. Como a área 1 é caracterizada por um trecho contínuo de mata, algumas espécies estão favorecidas pela maior disponibilidade de locais de nidificação, especialmente as que fazem seus ninhos em cavidades pré-existentes - como é o caso das abelhas sem ferrão, representadas pelos gêneros Plebeia Schwarz, 1938 e Xylocopa Latreille, 1802 e das abelhas da subtribo Euglossina. Na área 2, entretanto, por ser caracterizada por grandes afloramentos rochosos, ser uma área de uso extensivo (e conseqüentemente mais alterada), a vegetação é mais esparsa e com maior ocorrência de arbustos do que de árvores. Assim, representantes de Plebeia estão ausentes e as operárias de $T$. spinipes são muito mais raras do que na área 1.

A maior riqueza de espécies e abundância de indivíduos nas duas áreas de estudo foram encontradas em Apidae (Tab. III), devido ao comportamento eussocial dessas abelhas, à perenidade da colônia e aos hábitos generalizados de forrageio (RouBIK, 1989; MICHENER, 2000).

$\mathrm{Na}$ área de estudo, três espécies com comportamento eussocial representaram $78,2 \%$ do total de indivíduos coletados: A. mellifera, P. emerina e $T$. spinipes. Além das razões mencionadas por RoUBIK (1989), a grande abundância destas espécies eussociais também está relacionada à capacidade dessas abelhas em comunicar a localização das fontes de alimentos para as outras operárias da colônia, além de possuírem colônias populosas (Lindauer \& KerR, 1960), o que possibilita o aparecimento nas flores de um número elevado de abelhas provenientes de uma única colônia (SAKAGAMI et al., 1967).

Com cerca de $44,8 \%$ dos indivíduos, A. mellifera foi a espécie mais abundante nas duas áreas de estudo, com $39 \%$ do total de indivíduos coletados na área $1 \mathrm{e}$ $57 \%$ na área 2. Esse resultado é semelhante ao encontrado em muitos outros estudos realizados no Brasil (MARTINS, 1995; AgUiAR \& Martins, 1997; Viana et al., 1997; ViAnA, 1999; FARIA-MucCI et al., 2003; ZANElla, 2003; SANTOS et al., 2004). Segundo WiLms et al. (1996), o sucesso desta espécie introduzida e invasora pode ser explicado pelo fato que essas abelhas apresentam um maior nicho trófico do que as outras abelhas eussociais nativas. A diferença observada na abundância de A. mellifera entre as áreas

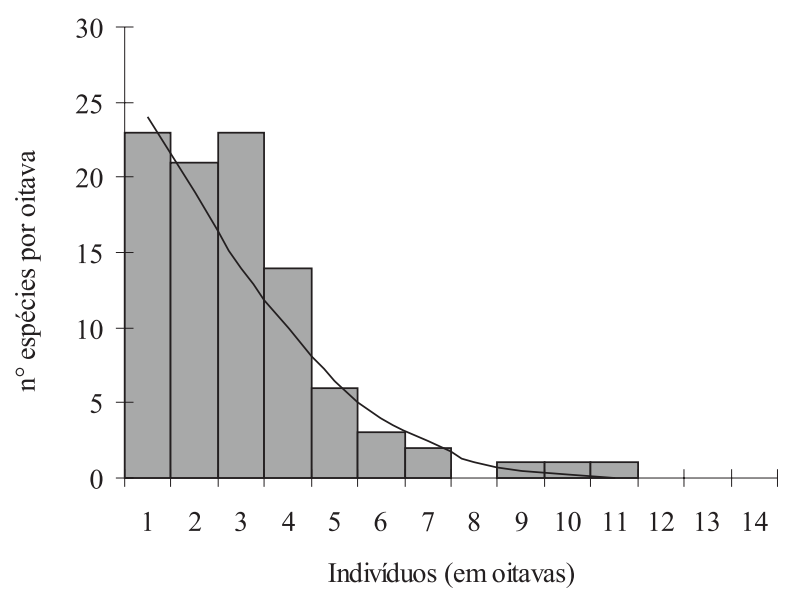

Fig. 1. Distribuição do número de espécies de Apoidea nas classes de abundância do número de indivíduos, em oitavas, no Parque Estadual de Itapuã, Viamão, RS, de agosto/2003 a julho/2004. 
Tabela I. Lista das espécies de abelhas amostradas nas duas áreas florestais do Parque Estadual de Itapuã, Viamão, RS, de agosto/2003 a julho/2004. A numeração das morfoespécies corresponde às numerações dos indivíduos da coleção de Insecta do Museu de Ciências e Tecnologia na Pontifícia Universidade Católica do Rio Grande do Sul, LPB-MCT/PUCRS.

\begin{tabular}{|c|c|c|c|c|c|}
\hline \multirow{2}{*}{ Espécies } & \multicolumn{2}{|c|}{ Área 1} & \multirow{2}{*}{\multicolumn{2}{|c|}{ Área 2}} & \multirow{2}{*}{ Total } \\
\hline & q & $0^{x}$ & & & \\
\hline
\end{tabular}

\section{Panurginae}

Calliopsini

Callonychium petuniae Cure \& Wittmann, 1990

Protandrenini

Anthrenoides micans Urban, 1995

Anthrenoides sp. 15

Anthrenoides sp. 16

Psaenythia bergi Holmberg, 1884

APIDAE

Apinae

Apini

Apina

Apis mellifera Linnaeus, 1758

Bombina

Bombus (Fervidobombus) atratus Franklin, 1913

Bombus (Fervidobombus) morio (Swederus, 1787)

Euglossina

Eufriesea violacea (Blanchard, 1840)

Euglossa (Euglossella) mandibularis Friese, 1899

Eulaema (Apeulaema) nigrita Lepeletier, 1841

Eulaema (Eulaema) nigrifascies (Friese, 1898)

Meliponina

Plebeia emerina (Friese, 1900)

Plebeia nigriceps (Friese, 1901)

Trigona spinipes (Fabricius, 1793)

Centridini

Centris (Hemisiella) tarsata Smith, 1874

Centris (Hemisiella) sp. 1

Centris (Trachina) fuscata Lepeletier, 1841

Epicharis (Hoplepicharis) affinis Smith, 1871

Emphorini

Melitoma segmentaria (Fabricius, 1804)

Eucerini

Alloscirtetica sp. 1

Thygater (Thygater) analis (Lepeletier, 1841)

Thygater (Thygater) sp. 2

Tapinotaspidini

Arhysoceble picta (Friese, 1899)

Lanthanomelissa clementis Urban, 1995

Paratetrapedia (Paratetrapedia) sp. 1

Tetrapediini

Tetrapedia diversipes Klug, 1810

Nomadinae

Nomadinae sp. 1

Nomadinae sp. 2

$\begin{array}{rr} & 24 \\ 5 & 3 \\ 3 & \\ 16 & \\ & \\ 337 & \\ 4 & \\ 563 & \end{array}$

Xylocopinae

Ceratinini

Ceratina (Ceratinula) muelleri Friese, 1910

Ceratina (Ceratinula) sclerops Schrottky, 1907

Ceratina (Ceratinula) sp. 8

Ceratina (Crewella) asuncionis Strand, 1910

Ceratina (Rhysoceratina) hyemalis Moure, 1950

Ceratina asunciana Strand, 1910

Ceratina richardsoniae Schrottky, 1909

Ceratina sp. 3

Xylocopini

Xylocopa (Neoxylocopa) frontalis (Olivier, 1789)

Xylocopa (Neoxylocopa) nigrocincta Smith, 1854

Xylocopa (Neoxylocopa) ordinaria Smith, 1874

Xylocopa (Stenoxylocopa) artifex Smith, 1874

COLLETIDAE

Colletinae

Colletes cf. furfuraceus Holmberg, 1886

\begin{tabular}{|c|c|c|c|}
\hline & & 1 & 1 \\
\hline 1 & & & \\
\hline \multirow[t]{3}{*}{9} & 1 & & \\
\hline & & 2 & 1 \\
\hline & & & 1 \\
\hline 1 & 1 & & \\
\hline 1 & & & \\
\hline 6 & 5 & & \\
\hline & & 1 & \\
\hline \multirow[t]{2}{*}{2} & & & \\
\hline & & 5 & 2 \\
\hline 2 & 2 & 1 & \\
\hline 1 & 1 & & \\
\hline 3 & & 6 & 2 \\
\hline 1 & & 3 & \\
\hline 3 & 1 & & 1 \\
\hline 3 & & 5 & 1 \\
\hline \multirow[t]{2}{*}{2} & 1 & 5 & 1 \\
\hline & & 26 & 13 \\
\hline 54 & 1 & & \\
\hline 1 & & 2 & \\
\hline 15 & & & \\
\hline 9 & & 3 & \\
\hline 1 & & & \\
\hline 2 & & & \\
\hline
\end{tabular}

6

1

10

3

1

2

11 


\begin{tabular}{|c|c|c|c|c|c|}
\hline \multicolumn{6}{|l|}{ Tabela I (cont.) } \\
\hline \multirow[t]{2}{*}{ Espécies } & \multicolumn{2}{|c|}{ Área 1} & \multicolumn{2}{|c|}{ Área 2} & \multirow[t]{2}{*}{ Total } \\
\hline & 우 & $0^{7}$ & 우 & $0^{7}$ & \\
\hline Colletes rugicollis Friese, 1900 & 4 & 1 & & & 5 \\
\hline Colletes sp. 9 & 1 & & & & 1 \\
\hline \multicolumn{6}{|l|}{ Diphaglossinae } \\
\hline \multicolumn{6}{|l|}{ Caupolicanini } \\
\hline Ptiloglossa cf. pretiosa (Friese, 1898) & 1 & & & & 1 \\
\hline \multicolumn{6}{|l|}{ Hylaeinae } \\
\hline Hylaeus rivalis (Schrottky, 1906) & 20 & & & & 20 \\
\hline Hylaeus sp. 4 & 1 & 1 & & & 2 \\
\hline Hylaeus sp. 16 & 6 & & & 1 & 7 \\
\hline Hylaeus sp. 18 & 2 & & & & 2 \\
\hline Hylaeus sp. 19 & 1 & & & & 1 \\
\hline Hylaeus sp. 20 & 2 & & & & 2 \\
\hline \multicolumn{6}{|l|}{ Paracolletinae } \\
\hline Cephalocolletes rugata Urban, 1995 & & & 4 & & 4 \\
\hline Perditomorpha sp. 6 & 1 & 4 & & & 5 \\
\hline \multicolumn{6}{|l|}{ HALICTIDAE } \\
\hline \multicolumn{6}{|l|}{ Halictinae } \\
\hline \multicolumn{6}{|l|}{ Augochlorini } \\
\hline Augochlora (Augochlora) amphitrite (Schrottky, 1910) & & & 4 & & 4 \\
\hline Augochlora (Augochlora) tantilla Moure, 1943 & 53 & 2 & 10 & 2 & 67 \\
\hline Augochlora (Augochlora) sp. 1 & 14 & & 2 & & 16 \\
\hline Augochlora (Augochlora) sp. 13 & 5 & & 1 & & 6 \\
\hline Augochlora (Oxystoglossella) semiramis (Schrottky, 1910) & 1 & 4 & 14 & 13 & 32 \\
\hline Augochlora (Oxystoglossella) sp. 1 & & 6 & & 1 & 7 \\
\hline Augochlora (Oxystoglossella) sp. 5 & 6 & & & & 6 \\
\hline Augochlorella ephyra (Schrottky, 1910) & 1 & & & & 1 \\
\hline Augochlorella michaelis (Vachal, 1911) & 4 & 3 & 1 & & 8 \\
\hline Augochlorella sp. 1 & & 1 & & & 1 \\
\hline Augochloropsis cupreola (Cockerell, 1900) & 1 & & 2 & & 3 \\
\hline Augochloropsis discors (Vachal, 1903) & 3 & 1 & & & 4 \\
\hline Augochloropsis euterpe (Holmberg, 1886) & & & 10 & 3 & 13 \\
\hline Augochloropsis sp. 6 & & & 3 & & 3 \\
\hline Augochloropsis sp. 20 & 3 & & & & 3 \\
\hline Augochloropsis sp. 21 & & & 1 & & 1 \\
\hline Neocorynura aenigma (Gribodo, 1894) & 12 & 6 & & 1 & 19 \\
\hline Paroxystoglossa brachycera (Moure, 1969) & 1 & & 1 & & 2 \\
\hline Pseudaugochlora graminea (Fabricius, 1804) & 7 & 1 & & & 8 \\
\hline Halictini & & & & & \\
\hline Dialictus anisitsianus (Strand, 1910) & 4 & & 2 & & 6 \\
\hline Dialictus travassosi (Moure, 1940) & 6 & & & & 6 \\
\hline Dialictus sp. 1 & 2 & & 3 & & 5 \\
\hline Dialictus sp. 4 & & & 1 & & 1 \\
\hline Dialictus sp. 5 & & & & 1 & 1 \\
\hline Dialictus sp. 9 & 3 & 1 & 48 & 18 & 70 \\
\hline Dialictus sp.17 & 13 & & & 2 & 15 \\
\hline MEGACHILIDAE & & & & & \\
\hline Megachilinae & & & & & \\
\hline Anthidiini & & & & & \\
\hline Anthodioctes claudii Urban, 1999 & & & 1 & & 1 \\
\hline Anthodioctes megachiloides Holmberg, 1903 & 1 & 1 & & & 2 \\
\hline Epanthidium paraguayensis (Schrottky, 1908) & 1 & & 1 & & 2 \\
\hline Hypanthidium divaricatum (Smith, 1854) & 9 & 13 & & & 22 \\
\hline Megachilini & & & & & \\
\hline Coelioxys (Glyptocoelioxys) pampeana Holmberg, 1887 & & & 4 & 1 & 5 \\
\hline Coelioxys (Rhynocoelioxys) zapoteka Cresson, 1878 & 1 & & & & 1 \\
\hline Coelioxys sp. 3 & & 1 & & & 1 \\
\hline Coelioxys sp. 9 & & 2 & & & 2 \\
\hline Coelioxys sp. 10 & 2 & & 1 & & 3 \\
\hline Megachile (Austromegachile) sp. 1 & 5 & 2 & 1 & & 8 \\
\hline Megachile (Austromegachile) sp. 2 & 2 & 1 & 2 & & 5 \\
\hline Megachile (Cressionella) sp. 1 & 1 & & & & 1 \\
\hline Megachile (Leptorachis) sp. 1 & 1 & & 1 & 1 & 3 \\
\hline Megachile (Moureapis) sp. 1 & & 5 & & 2 & 7 \\
\hline Megachile (Pseudocentron) sp. 1 & 5 & & 4 & & 9 \\
\hline Megachile (Pseudocentron) sp. 2 & & 1 & 5 & & 6 \\
\hline TOTAL & 2.129 & 98 & 1.007 & 72 & 3.306 \\
\hline
\end{tabular}


estudadas pode ser ainda um reflexo da proximidade da área 2 a sítios e fazendas nos arredores do Parque, onde muitos moradores mantinham caixas de A. mellifera para a comercialização do mel, antes da desapropriação total do Parque. Após a desapropriação, essas famílias se instalaram em áreas próximas à região do Parque e mantêm até hoje a criação dessas abelhas.

A pobreza de espécies de abelhas sem ferrão da subtribo Meliponina nas duas áreas de estudo parece ser uma característica da região estudada. WITTMANN \& HoFFMANN (1990) registraram para a região de Viamão somente quatro espécies de Meliponina, das quais duas foram observadas no Parque, sendo contabilizadas agora para a região cinco espécies. As abelhas eussociais nativas competem em muitos habitats com a introduzida abelha africanizada, frequientemente causando um efeito negativo na comunidade (PEDro \& CAMARGo, 1991; WiLms et al., 1996; Aguiar \& Martins, 2003).

Com relação às abelhas da subtribo Euglossina, foram encontradas quatro espécies, sendo os machos de Eufrisea violaceae (Blanchard, 1840) capturados com o uso das armadilhas com escatol, os machos de Euglossa mandibularis Friese, 1899 com $\beta$-ionone e as fêmeas desta espécie diretamente nas flores (assim como todas as fêmeas das duas espécies de Eulaema). A diversidade de abelhas de Euglossina na região de estudo é relativamente alta quando comparada a outras regiões do estado. Seis espécies dessa subtribo foram encontradas no noroeste por Alves-DOS-SANTOS (1999), duas espécies na região do Planalto das Araucárias (dados não publicados) e duas em Guaíba por Wittmann \& HoffMann (1990). Segundo RoubIK (1989), abelhas dessa tribo estão mais ligadas a áreas de mata e o número de espécies da região sudeste para o sul diminui drasticamente.

Halictidae é a segunda família melhor representada em número de espécies $(27,4 \%)$ e indivíduos $(9,3 \%$, Tab. I, III) mostrando abundância e riqueza de espécies semelhantes nas duas áreas. Entretanto, a riqueza de espécies desta família no Parque é relativamente baixa quando comparada a outros estudos realizados no Rio Grande do Sul (Wittmann \& Hoffmann, 1990), onde a maior riqueza de espécies foi observada na região noroeste do Estado (82), seguida da região do Planalto das Araucárias (72) e região das Guaritas (58 espécies registradas) (Schlindwein, 1995; Alves-DOS-SANTOS, 1999).
Apesar de Megachilidae ser numerosa no Rio Grande do Sul (Alves-DOS-SANTOS, 1999), essa família não mostrou índices muito altos de abundância e riqueza na região estudada (78 indivíduos e 16 espécies) (Tab. I, III). O baixo número observado de espécies é semelhante ao encontrado por WitTMANn \& HoFFMANn (1990) numa área agrícola em Viamão. Entretanto, a riqueza de espécies desta família é bem maior nos trabalhos de SCHLINDWEIN (1995) e Alves-DOS-SAnTos (1999). RoubiK (1989) sugere que o menor número de espécies de Megachilidae em alguns locais deve-se ao fato de tratam-se de abelhas solitárias (com populações menos numerosas do que as de espécies sociais, subsociais e semisociais). Desta maneira, estas abelhas são mais raras e sazonais, dificultando a sua coleta nas flores.

Em termos de abundância e riqueza de espécies de Colletidae, destacou-se a riqueza da subfamília Hylaeinae, principalmente na área 1. Colletidae está bem representada no Rio Grande do Sul, sendo amplamente distribuída nas diversas regiões do estado (Schlindwein, 1995; AlvesDos-S Antos, 1999). Entretanto, até o momento, os registros de espécies de Hylaeinae limitaram-se para a região do Planalto das Araucárias (dados não publicados) e para a região das Guaritas (SCHLINDWEIN, 1995).

Dos levantamentos realizados no RS, o presente estudo foi o que registrou menor riqueza de abelhas da família Andrenidae (5 espécies, Tab. I, III), comparado com Wittmann \& Hoffmann (1990) (17), Schlindwein (1995) (28) e Alves-Dos-SAntos (1999) (26). Tal baixa diversidade registrada pode ser um reflexo da estreita relação dessas abelhas com determinadas espécies vegetais, principalmente com as espécies de hábito herbáceo (SCHLindwein, 1998), de pouca representatividade nas áreas amostradas.

Sazonalidade. O período de maior atividade de Apoidea ocorreu durante a primavera e o verão. Neste período, foram capturados $70 \%$ dos indivíduos e $81 \%$ das espécies (Figs. 2, 3). Representantes de Apidae e Halictidae foram encontrados o ano todo nas flores. $\mathrm{O}$ maior número de indivíduos de Apidae foi registrado no mês de setembro (466) e de espécies nos meses de janeiro e fevereiro (17). Halictidae apresentou um maior número tanto de indivíduos quanto de espécies nos meses de dezembro e janeiro. Megachilidae e Colletidae foram registradas somente nos meses de primavera e verão. $\mathrm{O}$

Tabela II. Comparação da diversidade de abelhas em estudos realizados ao longo de um ano em diferentes regiões do Brasil que utilizaram a mesma metodologia. Todos os estudos incluíram Apis mellifera nas coletas. (*dados referentes ao período entre 1992 e 1993 ; EA/h, Esforço amostral em horas; H', índice de Shannon-Wiener; J', equiitatividade).

\begin{tabular}{|c|c|c|c|c|c|c|c|}
\hline Autor & Estado & Vegetação & $\mathrm{EA} / \mathrm{h}$ & $\mathrm{n}^{\circ}$ espécies & $\mathrm{n}^{\circ}$ indivíduos & $\mathrm{H}^{\prime}$ & $\mathrm{J}^{\prime}$ \\
\hline Este estudo & RS & Floresta Estacional & 480 & 95 & 3.306 & 2,09 & 0,46 \\
\hline TAURA \& LAROCA $(2001)^{*}$ & PR & Área jardinada & 120 & 49 & 1.700 & 2,99 & 0,53 \\
\hline FARIA-MuCCI et al. (2003) & MG & Campo rupestre & 288 & 72 & 746 & 3,11 & 0,72 \\
\hline MARTINS (1994) & $\mathrm{BA}$ & Caatinga & 384 & 42 & 1.249 & 2,11 & 0,56 \\
\hline MARTINS (1994) & BA & Cerrado & 384 & 147 & 1.761 & 3,53 & 0,70 \\
\hline VIANA (1999) & $\mathrm{BA}$ & Dunas & 312 & 31 & 931 & 2,25 & 0,65 \\
\hline SANTOS et al. (2004) & TO & Cerrado/Amazônia & 624 & 83 & 5.534 & 1,55 & 0,35 \\
\hline AgUiAR \& MARTINS (1997) & $\mathrm{PB}$ & Caatinga & 384 & 45 & 950 & 2,32 & 0,61 \\
\hline AgUiAR \& MARTINS (2003) & $\mathrm{PB}$ & Caatinga/ Cerrado/ Mata Atlântica & 403 & 114 & 3.022 & 3,37 & 0,71 \\
\hline ZANELLA (2003) & $\mathrm{RN}$ & Caatinga & 192 & 100 & 3.164 & 2,42 & 0,52 \\
\hline
\end{tabular}




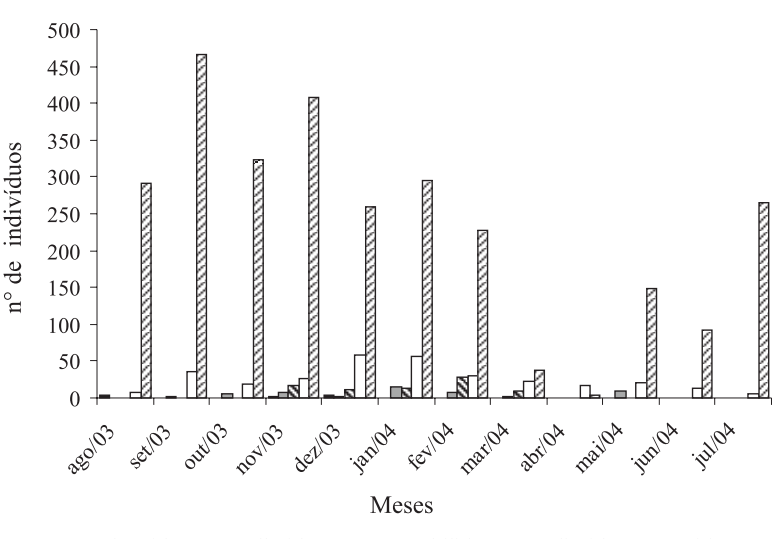

Andrenidae $\square$ Colletidae Megachilidae $\square$ Halictidae $\measuredangle$ Apidae

Fig. 2. Variação sazonal do número de indivíduos de abelhas em atividade, ordenados por família, no Parque Estadual de Itapuã, Viamão, RS, de agosto/2003 a julho/2004.

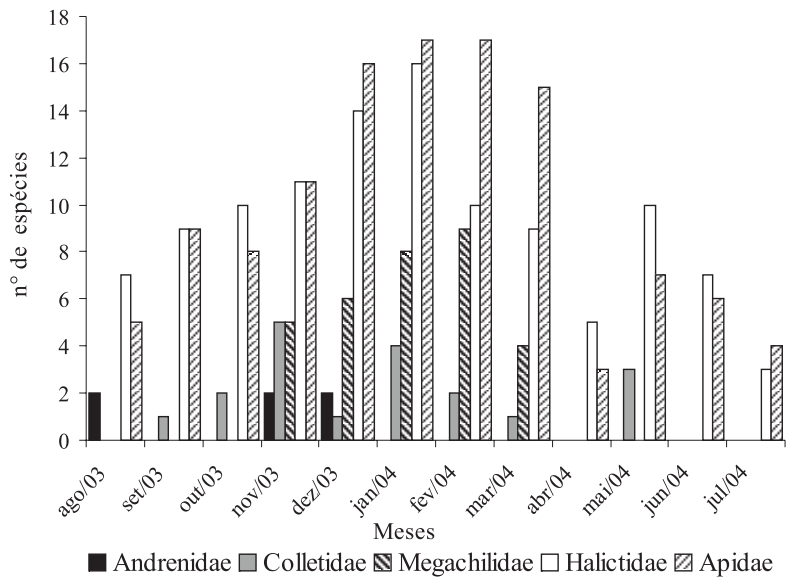

Fig. 3. Variação sazonal do número de espécies de abelhas em atividade, ordenadas por família, no Parque Estadual de Itapuã, Viamão, RS, de agosto/2003 a julho/2004.

Tabela III. Freqüência absoluta e relativa de espécies $(n=95)$ e indivíduos $(n=3.306)$ de abelhas coletadas por família, nas duas áreas de estudo no Parque Estadual de Itapuã, Viamão, RS, de agosto/2003 a julho/2004.

\begin{tabular}{lcccc}
\hline & \multicolumn{3}{c}{ Área 2} \\
\cline { 2 - 5 } Família & $\mathrm{n}^{\circ}$ espécies $(\%)$ & $\mathrm{n}^{\circ}$ indivíduos $(\%)$ & $\mathrm{n}^{\circ}$ espécies $(\%)$ & $\mathrm{n}^{\circ}$ indivíduos $(\%)$ \\
\hline Andrenidae & $3(3,1)$ & $4(0,1)$ & $2(2,1)$ & $5(0,2)$ \\
Apidae & $29(30,5)$ & $1.959(59,2)$ & $19(20)$ & $901(27,2)$ \\
Colletidae & $11(11,6)$ & $46(1,4)$ & $2(2,1)$ & $5(0,2)$ \\
Halictidae & $20(21,1)$ & $164(5)$ & $19(20)$ & $144(4,3)$ \\
Megachilidae & $14(14,7)$ & $54(1,6)$ & $10(10,5)$ & $24(0,7)$ \\
\hline TOTAL & $77(81)$ & $2.227(67,3)$ & $52(54,7)$ & $1.079(32,6)$ \\
\hline
\end{tabular}

número máximo de indivíduos (28) e de espécies (nove) da família Megachilidae foi registrado no mês de fevereiro. Para Colletidae, o número máximo de espécies foi registrado em novembro (cinco) e de indivíduos em janeiro (15) (Figs. 2, 3). Abelhas da família Andrenidae foram amostradas somente nos meses de agosto, novembro e dezembro, com um número muito baixo tanto de espécies quanto de indivíduos.

O declínio generalizado no número de espécies e indivíduos observado durante o mês de abril, provavelmente está associado com a diminuição da produção de flores, decorrente da baixa pluviosidade e alta temperatura. Segundo AgUiAR \& MARTINs (1997), a ocorrência de chuvas influência diretamente os padrões de florescimento das plantas e, conseqüentemente, a disponibilidade de recursos alimentares para as abelhas. Porém, no caso das abelhas solitárias, provavelmente, este declínio deve-se ao fato de que apresentam ciclo de vida extremamente sazonal, passando os meses de outono e inverno em diapausa (MANSINGH, 1971).

Agradecimentos. Agradecemos a Mardiore Pinheiro do Instituto de Biologia da UNICAMP pelo acesso à bibliografia, aos alunos da PUCRS Gabriela S. Franco, Cesar C. Jung, Sarina Held, ao amigo Pedro Paulo Marques pelo auxílio nas coletas e trabalho de laboratório e ao Prof. Dr. Wolf Engels da Universidade de Tübingen, Alemanha, pela liberação dos veículos para as viagens a campo. Agradecemos ainda ao $\mathrm{CNPq}$ pela bolsa concedida à primeira autora.

\section{REFERÊNCIAS BIBLIOGRÁFICAS}

Aguiar, C. M. L. 2003. Utilização de recursos florais por abelhas (Hymenoptera, Apoidea) em uma área de Caatinga (Itatim, Bahia, Brasil). Revista Brasileira de Zoologia 20(3):457-467.

Aguiar, C. M. L. \& Martins, C. E. 1997. Abundância relativa, diversidade e fenologia de abelhas (Hymenoptera, Apoidea) na caatinga, São João do Cariri, Paraíba, Brasil. Iheringia, Série Zoologia, 83:151-163.

2003. The bee diversity of the Tabuleiro vegetation in the Guaribas Biological Reserve (Mamanguape, Paraíba, Brazil). In: Melo, G. A. R. \& Alves-dos-Santos, I. eds. Apoidea Neotropica: homenagem aos 90 anos de Jesus Santiago Moure. Criciúma, UNESC. p.209-216.

Alves-dos-Santos, I. 1999. Abelhas e plantas melíferas da Mata Atlântica, Restinga e Dunas do litoral norte do Estado do Rio Grande do Sul, Brasil. Revista Brasileira de Entomologia 43(3/4): 191-223.

Antonini, Y. \& Martins, R. P. 2003. The flowering-visiting bees at the Ecological Station of the Universidade Federal de Minas Gerais, Belo Horizonte, MG, Brazil. Neotropical Entomology 32(4):565-575.

Batra, S. W. 1984. Solitary bees. Scientific American 250(2):86-93.

BAwA, K. S. 1990. Plant-Pollinator Interactions in Tropical Rain Forests. Annual Review of Ecology and Systematics 21:399-422.

Brack, P.; Rodrigues, R. S. \& Leite, S. L. C. 1998. Morro do Osso - um santuário cercado pela cidade. In: Menegat, R. ed. Atlas ambiental de Porto Alegre. Porto Alegre, Universidade Federal do Rio Grande do Sul. p.80.

Camargo, J. M. F. \& Mazucato, M. 1984. Inventário da apifauna e flora apícola de Ribeirão Preto, SP, Brasil. Dusenia 14(2):55-87. 
Campos, L. A. O.; Silveira F. A. da; Oliveira, M. L. de; Abrantes, C. V. M.; Morato, E. F. \& Melo, G. A. R. DE. 1989. Utilização de armadilhas para captura de machos de Euglossini (Hymenoptera, Apoidea). Revista Brasileira de Zoologia 6(4):621-626.

Cockerell, T. D. A. 1900. Descriptions of new bees collected by H. H. Smith in Brazil. Proceedings of the Academy of Natural Sciences of Philadelphia 52:336-337.

Ducke, A. 1906. Neue Beobachtungen über die Bienen der Amazonasländer. Allgemeine Zeitschrift für Entomologie 2:51-60.

1907. Contribution à la connaissance de la faune hyménoptèrologique du Nordest du Brésil. Revue d'Entomologie 26:73-96.

1925. Die stachellosen Bienen Brasiliens. Zoologische Jahrbücher - Abteilung für Systematik, Ökologie und Geographie der Tiere 49:335-448.

Faria-Mucci, G. M; Melo, M. A. \& Campos, L. A. 2003. A fauna de abelhas (Hymenoptera, Apoidea) e plantas utilizadas como fonte de recursos florais, em um ecossistema de campos rupestres em Lavras, Minas Gerais, Brasil. In: Melo, G. A. R. \& Alves-dos-Santos, I. eds. Apoidea Neotropica: homenagem aos 90 anos de Jesus Santiago Moure. Criciúma, UNESC. p.241-256.

Friese, H. 1910. Neue Bienenarten aus Süd-Amerika. Deutsche Entomologische Zeitschrift 56:693-711.

Gottsberger, G.; Camargo, J. M. F.; Silberbauer-Gottsberger, I. 1988. A bee pollinated tropical community: the beach dune vegetation of Ilha de São Luís, Maranhão, Brazil. Botanische Jahrbücher für Systematik, Pflanzengeschichte und Pflanzengeographie 109(4):469-500.

Kevan, P. G. \& BAKeR, H. G. 1983. Insects as flower visitors and pollinators. Annual Review of Entomology 28:407-453.

Krebs, C. J. 1998. Ecological Methodology. New York, Addison Wesley Longman. 620p.

LAROCA, S. 1992. Community ecology in bees: relative importance of rare and common species in some holarctic and neotropical sites. Revista Brasileira de Zoologia 9(1/2):131-137.

Lindauer, M. \& KerR, W. E. 1960. Communication between workers of stingless bees. Bee World 41(2):29-41.

Mansingh, A. 1971. Physiological classification of dormancies in insects. The Canadian Entomologist 121: 745-756.

Martins, C. F. 1994. Comunidade de abelhas (Hym., Apoidea) da caatinga e do cerrado com elementos de campo rupestre do Estado da Bahia, Brasil. Revista Nordestina de Biologia 9(2):225-257.

1995. Flora apícola e nichos tróficos de abelhas (Hym., Apoidea) na Chapada Diamantina (Lençóis, BA, Brasil). Revista Nordestina de Biologia 10(2):119-140.

Matheson, A.; Buchmann, S. L.; O'toole, C.; Westrich, P. \& Williams, I. H. 1996. The conservation of bees. London, Academic. 254p.

Michener, C. D. 2000. The bees of the World. Baltimore, Johns Hopkins University. 913p.

Palma, C. B. \& Jarenkow, J. A. 2003. Efeito da sazonalidade na estrutura da sinúsia herbácea terrícola de uma floresta estacional, Viamão, RS, In: Claudino-Sales, V; Tonini, I. M. \& Dantas, E. W. C. eds. Ecossistemas Brasileiros - manejo e conservação (Simpósios). Fortaleza, Universidade Federal do Ceará. p.413-415.

Pedro, S. R. M. \& Camargo, J. M. F. 1991. Interactions on floral resources between the Africanized honey bee Apis mellifera L. and the native bee community (Hymenoptera: Apoidea) in natural "cerrado" ecosystem in southeast Brazil. Apidologie 22(4):397-415

Porto, M. L. \& Mello, R. S. P. 1998. Mapa da vegetação natural atual. In: Menegat, R. ed. Atlas ambiental de Porto Alegre. Porto Alegre, Universidade Federal do Rio Grande do Sul. p.53-55.

Preston, F. W. 1948. The commonness and rarity of species. Ecology 29(3):254-283.
Proctor, M.; Yeo, P. \& Lack, A. 1996. The natural history of pollination. London, Harper Collins. 479p.

RADAMBRASIL, 1981. Levantamento de Recursos Naturais, v. 19, Folha SD 22. Rio de Janeiro, Projeto Radambrasil. 624p.

Rio Grande do Sul. 1997. Plano de Manejo: Parque Estadual de Itapuã. Porto Alegre, Departamento de Recursos Naturais Renováveis. $158 \mathrm{p}$.

RouвiK, D. W. 1989. Ecology and natural history of tropical bees. Cambridge, Cambridge University. 514p.

SaKagami, S. F. \& Laroca, S. 1971. Relative abundance, phenology and flower visits of apid bees in eastern Paraná, southern Brazil (Hymenoptera, Apidae). Kontyû 39(3):217-230.

Sakagami, S. F. \& Matsumura, T. 1967. Relative abundance, phenology and flower preference of andrenid bees in Sapporo, North Japan (Hymenoptera, Apoidea). Japanese Journal of Ecology 17(6):237-250.

Sakagami, S. F.; Laroca, S. \& Moure, J. S. 1967. Wild bees biocenotics in São José dos Pinhais (PR), South Brazil preliminary report. Journal of the Faculty of Science Hokkaido University 16(2):253-291.

Santos, F. M.; Carvalho, C.; A. L. \& Silva, R. F. 2004. Diversidade de abelhas (Hymenoptera: Apoidea) em uma área de transição Cerrado-Amazônia. Acta Amazonica 34(2):319-328.

Schlindwein, C. 1995. Wildbienen und ihre Trachtpflanzen in einer südbrasilianischen Buschlandschaft: Fallstudie Guaritas, Bestäubung bei Kakteen und Loasaceen. Stuttgart, Ulrich Grauer. 148p.

1998. Frequent oligolecty characterizing a diverse beeplant community in a xerophytic bushland of subtropical Brazil. Studies on Neotropical Fauna and Environment 33(1):46-59.

Schrottкy, C. 1902. Ensaio sobre abelhas do Brasil. Revista do Museu Paulista 5:330-613.

Silveira, F. A.; Melo, G. A. R. \& Almeida, E. A. B. 2002. Abelhas brasileiras: sistemática e identificação. Belo Horizonte, Fundação Araucária. 253p.

Taura, H. M. \& Laroca, S. 2001. A associação de abelhas silvestres de um biótopo urbano de Curitiba (Brasil), com comparações espaço-temporais: abundância relativa, fenologia, diversidade e explotação de recursos (Hymenoptera, Apoidea). Acta Biologica Paranaense 30(1-4):35-137.

Teixeira, M. B.; Coura-Neto, A. B.; Pastore, U. \& Rangel-Filho, A. L. R. 1986. Vegetação - as regiões fitoecológicas, sua natureza e seus recursos econômicos. Estudo fitogeográfico. In: PROJETO RadAmbrasil. Levantamento de Recursos Naturais. Rio de Janeiro, IBGE. v. 33, p.541-632.

VianA, B. F. 1999. A comunidade de abelhas (Hymenoptera: Apoidea) das Dunas interiores do Rio São Francisco, Bahia, Brasil. Anais da Sociedade Entomológica do Brasil 28(4):635-645.

Viana, B. F.; Kleinert, A. M. P. \& Imperatriz-Fonseca, V. L. 1997. Abundance and flower visits of bees in cerrado of Bahia, tropical Brazil. Studies on Neotropical Fauna and Environment 32(4):212-219.

Williams, I. H.; Corbet, S. A. \& Osborne, J. L. 1991. Beekeeping, wild bees and pollination in the European Community. Bee World 72(4): 170-180.

WiLMS, W.; IMPERATRIZ-FonseCA, V. L \& ENGELS W. 1996. Resource partitioning between highly eusocial bees and possible impact of the introduced Africanized honey bee on native stingless bees in the Brazilian Atlantic Rainforest. Studies on Neotropical Fauna and Environment 31(3/4):137-151.

Wittmann, D. \& Hoffmann, M. 1990. Bees of Rio Grande do Sul, southern Brazil (Insecta, Hymenoptera, Apoidea). Iheringia, Série Zoologia, 70:17-43.

Zanella, F. C. V. 2003. Abelhas da Estação Ecológica do Seridó (Serra Negra do Norte, RN): aportes ao conhecimento da diversidade, abundância e distribuição espacial das espécies na caatinga. In: Melo, G. A. R. \& Alves-Dos-Santos, I. eds. Apoidea Neotropica: homenagem aos 90 anos de Jesus Santiago Moure. Criciúma, UNESC. p.231-240. 